\title{
The Symptoms and Clinical Manifestations Observed in COVID-19 Patients/Long COVID-19 Symptoms that Parallel Toxoplasma gondii Infections
}

\author{
Kevin $\operatorname{Roe}^{1}$ (D) \\ Received: 29 March 2021 / Accepted: 11 May 2021 / Published online: 29 May 2021 \\ (c) The Author(s), under exclusive licence to Springer Science+Business Media, LLC, part of Springer Nature 2021
}

Keywords Brain Infections · Latent Infections · Protozoa · Protozoan Infections · SARS-CoV-2 Mortality

Several peculiar symptoms and subpopulation mortality rates have been documented in COVID-19 patients and in patients manifesting the post-infection symptoms now called Long COVID-19 (Carvalho-Schneider et al. 2020; Yelin et al. 2020; Mendelson et al. 2020; Nemani et al. 2021; Kozloff et al. 2020; Zheng et al. 2020a, b). As an example, in a study of 293 reverse transcriptasePCR test confirmed COVID-19 hospital inpatients and outpatients in Tours, France, even after excluding 64 critical disease ICU patients and 36 residents transferred to a nursing home or long-term care facility, there were still 19 deaths (Carvalho-Schneider et al. 2020). In a followup study of 130 recovered non-critical COVID-19 patients at the same hospital, over $22 \%$ of the patients on day 60 after symptom onset reported the unusual symptoms of anosmia (impaired smell) and ageusia (impaired taste), and over $7 \%$ still reported the symptoms of dyspnoea (shortness of breath) (Carvalho-Schneider et al. (2020). Over $33 \%$ of these recovered COVID-19 patients reported that they felt as bad on day 60 after symptom onset as they felt during their acute disease phase (Carvalho-Schneider et al. 2020). One explanatory hypothesis would be that for some individuals these peculiar symptoms and subpopulation mortality rates are the consequence of SARS-CoV-2 virus infections working in bidirectional synergy with one or more existing latent pathogen infections, by means of induced mutually beneficial immune cell dysfunctions, including T cell exhaustion (Zheng et al. 2020a, b; Varikuti et al. 2018; Xiao et al. 2018). Based on medical observations, the most plausible participating latent pathogen

Kevin Roe

kevin.roe@att.net

1 San Jose, CA, USA infection is the wide-spread protozoan parasite Toxoplasma gondii (Varikuti et al. 2018; Xiao et al. 2018).

Protozoan parasites, including Toxoplasma gondii, cause intracellular infections in a large percentage of the global human population (Varikuti et al. 2018; Xiao et al. 2018). T. gondii has three life cycles, including tachyzoites that cause acute infection, bradyzoite tissue cysts that maintain a latent infection, and sporozoites that infect new hosts by food or water (Xiao et al. 2018). Other than by occasional T. gondii transmissions by organ or tissue transplant procedures, a new host is usually infected after ingestion of sporozoites allowing activated protozoa to penetrate the gut epithelium and reach the host's bloodstream (Xiao et al. 2018).

Protozoan parasites, including T. gondii, can latently infect the brain and central nervous system (CNS), leaving tissue cysts in hosts and immune system dysfunctions (Xiao et al. 2018). T. gondii has been extensively linked to numerous mental illnesses, including recent onset psychiatric illnesses, such as schizophrenia, in some individuals (Xiao et al. 2018).

T. gondii bradyzoites have been detected in muscles, brain neurons, and the CNS including the retina of the eye (Xiao et al. 2018). Humans with brain infections by $T$. gondii present a neuroinflammation of the brain, activated microglia and astrocytes in the brain, and complement factor C1q near the cysts (Xiao et al. 2018). Furthermore, it has been shown that $T$. gondii cysts in the brain induce major disruptions in the levels of glutamate, gamma-aminobutyric acid, dopamine and other neurotransmitters (Xiao et al. 2018). It has been asserted that such neurotransmitter disruptions also induce schizophrenia, particularly in cases of recent onset of schizophrenia (Xiao et al. 2018). In fact, a statistically significant link between schizophrenia and a higher mortality rate from SARS-CoV-2 has been reported, with a mortality odds ratio of 2.67 compared to reference patients (Nemani 
et al. 2021). This could result from even a small percentage of such patients having schizophrenia caused by $T$. gondii cysts .

Infected hosts have also demonstrated CD8 $\mathrm{T}$ cell exhaustion, which is believed to ultimately induce $T$. gondii reactivations and localized inflammations (Xiao et al. 2018). It is particularly noteworthy that $\mathrm{T}$ cell exhaustion, such as CD8 $T$ cell exhaustion, and a reduced host antiviral response have also been seen in COVID-19 patients who exhibit worse infections and worse outcomes (Zheng et al. 2020a, b).

\section{An Explanation for Higher COVID-19 Mortality in Other Categories}

COVID-19 has several distinctive and peculiar hospitalization and mortality characteristics (Gottlieb et al. 2020; Harrison et al. 2020; Hamer et al. 2020). In a large studies of 31,461 adults in the U.S., and 334,329 patients in the U.K., COVID19 patients had increasing hospitalization rates for increasingly older adults (adjusted odds ratios of .92 for 0-18 year age group, 1.0 for 19-44, 1.67 for 45-54, 1.99 for 55-64, 4.55 for 65-74, and 7.32 for adults over 75), a progressively higher mortality rate for older adults (adjusted odds ratio ranging from 1.06 to 1.08 per extra year of age), a higher mortality rate of males compared to females (adjusted odds ratio ranging from 1.6 to 1.75 compared to females), and a higher mortality rate for patients reaching body mass indexes of 30 to 35 (adjusted odds ratio ranging from 1.2 to 1.37) (Gottlieb et al. 2020; Harrison et al. 2020; Hamer et al. 2020).

There are significant observational similarities between the mortality rates of the preceding categories and their latent pathogen infection rates with protozoan parasites like $T$. gondii (Harrison et al. 2020; Hamer et al. 2020; Wilking et al. 2016). In Germany in 2016, the seroprevalence of $\operatorname{IgG}$ antibodies to $T$. gondii in 6,663 adults ranging in age 18 to 79 progressively increased from $20 \%$ in the 18 to 29 age group to $77 \%$ in the 70 to 79 age group, where males had higher infection rates than females (an odds ratio of 1.8 for males), and higher infection rates for adults with body mass indexes over 30 (with an odds ratio of 1.3) (Wilking et al. 2016). If $T$. gondii infection rates are approximately equivalent among relatively high income countries (e.g., the U.S., the U.K. and Germany), the close matches between increased COVID-19 mortality rates and $T$. gondii infection rates in the same corresponding subpopulations could be logically explained by the involvement of a mutually beneficial immune system dysfunction, such as CD8 T cell exhaustion, assisting both COVID-19 and $T$. gondii infections. This synergy could explain a large number of the peculiar aspects of COVID-19. But this synergy could also explain many aspects of Long COVID-19.

\section{An Explanation for Long COVID-19}

Latent infections, including T. gondii infections, can explain the increased mortality rates for several categories of COVID-19 patients. Such latent infections can also explain why several patients suffer severe COVID-19 symptoms, and explain why several patients suffer "Long COVID-19" (Carvalho-Schneider et al. 2020; Yelin et al. 2020; Mendelson et al. 2020). A large fraction of patients, after their COVID-19 viral infections, suffer several neurological and psychological symptoms of Long COVID-19 for several months, including: fatigue, dyspnoea (labored breathing/shortness of breath), anosmia (impaired sense of smell), dysgeusia (impaired sense of taste), chest pain, joint pain, hair loss, memory and attention deficits, anxiety, depression, and sleep disorders, (Carvalho-Schneider et al. 2020; Yelin et al. 2020; Mendelson et al. 2020).

It is significant that virtually all of the diverse and distinctive symptoms of Long COVID-19 can be explained as symptoms of toxoplasmosis in patients having activated or reactivated T. gondii (Vidal 2019). Toxoplasmosis patients suffer a wide variety of symptoms, including dyspnoea, fevers, seizures, headaches, changes in vision, altered mental status, focal neurological deficits, mental confusion, cognitive dysfunction, ataxia, behavioral or psychomotor changes, involuntary movements, pneumonia, and a spectrum of cranial nerve palsies (Vidal 2019).

Several distinctive Long COVID-19 symptoms can be matched if toxoplasmosis induced cranial nerve palsies affect the functions of the 12 cranial nerves, creating symptoms including anosmia from a palsy of the olfactory nerve (I), vertigo (dizziness), tinnitus and otalgia (earache) from a palsy of the vestibulocochlear nerve (VIII), and dysgeusia from palsies of the facial nerve (VII), glossopharyngeal nerve (IX), and vagus nerve (X) (Khaku et al. 2017).

Table 1 lists the symptoms of toxoplasmosis versus the symptoms of COVID-19 and/or Long COVID-19. Toxoplasmosis symptoms can match several symptoms exhibited in COVID-19 and/or Long COVID-19 patients.

In summary, protozoan parasite brain infections by $T$. gondii have been observed to cause neuroinflammations, neurotransmitter disruptions, and immune dysfunctions, such as CD8 T cell exhaustion. Such infections have been linked to some cases of schizophrenia. Observations of an increased SARS-CoV-2 mortality in schizophrenia patients can be explained by some schizophrenia patients exhibiting $T$. gondii brain infections that induce neuroinflammation and $\mathrm{T}$ cell exhaustion, such as CD8 $\mathrm{T}$ cell exhaustion, that assist the SARS-CoV-2 virus. Furthermore, the very close matches between COVID-19 mortality rates and $T$. 
Table 1 The Symptoms of Toxoplasmosis Versus the Symptoms of COVID-19 and/or Long COVID-19-References (Carvalho-Schneider et al. 2020; Yelin et al. 2020; Mendelson et al. 2020; Wilking et al. 2016; Vidal 2019; Khaku et al. 2017)

\begin{tabular}{llll}
\hline Symptom & Toxoplasmosis & COVID-19 & Comment \\
\hline Fatigue & Documented & Documented & Definitely seen in both \\
Dyspnoea/Labored breathing & Documented & Documented & Definitely seen in both \\
Fever & Documented & Documented & Definitely seen in both \\
Altered Mental Status ${ }^{\text {a }}$ & Documented & Documented & Definitely seen in both \\
Mental Confusion ${ }^{\text {a }}$ & Documented & Documented & Definitely seen in both \\
Cognitive Dysfunction ${ }^{\text {a }}$ & Documented & Documented & Definitely seen in both \\
Behavioral Changes $^{\text {a }}$ & Documented & Documented & Definitely seen in both \\
Neurological Deficits & Documented & Documented & Definitely seen in both \\
Ataxia/Incoordination* & Documented & Documented & Definitely seen in both \\
Involuntary Movements* & Documented & Documented & Definitely seen in both \\
Anosmia/Impaired smell & Possible & Documented & T. gondii C.N. palsy induced \\
Dysgeusia/Impaired taste & Possible & Documented & T. gondii C.N. palsy induced \\
Chest Pain & Possible & Documented & T. gondii nerve palsy induced \\
Arthralgia/Joint pain & Possible & Documented & T. gondii nerve palsy induced \\
Myalgia/Muscle pain & Possible & Documented & T. gondii nerve palsy induced \\
Hair Loss & Possible & Documented & Resultant stress could do this \\
Anxiety & Possible & Documented & Resultant stress could do this \\
Depression & Possible & Documented & Resultant stress could do this \\
Seizures & Documented & Possible & Seen in COVID-19 \\
Headaches & Documented & Possible & Seen in COVID-19 \\
Vision Changes & Documented & Possible & Seen in COVID-19 \\
Pneumonia & Documented & Documented & Seen in COVID-19 \\
\hline
\end{tabular}

C. N. palsy means cranial nerve palsy

*Ataxia (Incoordination or lack of coordination) and Involuntary Movements are broad symptoms and may be documented with overlaps

${ }^{a}$ Altered Mental Status, Mental Confusion, Cognitive Dysfunction, and Behavioral Changes are broad symptoms and may be documented with overlaps gondii infection rates in several of the same corresponding subpopulation categories imply that latent $T$. gondii infections can explain several unusual mortality aspects of COVID-19. Finally, the partial or full reactivation of latent $T$. gondii infections can explain why many individuals exhibit a very diverse set of symptoms of Long COVID19 well after the end of their SARS-CoV-2 infections.

Acknowledgements There are no acknowledgments.

Funding This research did not receive any specific grant from funding agencies in the public, commercial, or not-for-profit sectors.

Data Availability Data sharing is not applicable to this letter as no new data were created or analyzed in this study.

\section{Declarations}

Ethics Approval The author confirms that the ethical policies of the journal, as noted on the journal's author guidelines page, have been adhered to. No ethical approval was required as this is a letter with no original research data.

Conflict of Interest The author has no potential conflicts of interest.

\section{References}

Carvalho-Schneider C, Laurent E, Lemaignen A, Beaufils E, BourbaoTournois C, Laribi S, Flament T et al (2020) Follow-up of adults with non-critical COVID-19 two months after symptoms' onset. Clin Microbiol Infect. https://doi.org/10.1016/j.cmi.2020.09.052

Gottlieb M, Sansom S, Frankenberger C, Ward E, Hota B (2020) Clinical course and factors associated with hospitalization and critical illness among COVID-19 patients in Chicago, Illinois . Acad Emerg Med 27(10):963-973. https://doi.org/10.1111/acem.14104

Hamer M, Gale CR, Kivimäki M, Batty GD (2020) Overweight, obesity, and risk of hospitalization for COVID-19: a communitybased cohort study of adults in the United Kingdom. Proc Natl Acad Sci USA 117(35):21011-21013

Harrison SL, Fazio-Eynullayeva E, Lane DA, Underhill P, Lip GYH (2020) Comorbidities associated with mortality in 31,461 adults with COVID-19 in the United States: a federated electronic medical record analysis. PLoS Med 17(9):e1003321

Khaku A, Patel V, Zacharia T, Goldenberg D, McGinn J (2017) Guidelines for radiographic imaging of cranial neuropathies. Ear Nose Throat J 96(10-11):E23-E39

Kozloff N, Mulsant BH, Stergiopoulos V, Voineskos AN (2020) The COVID-19 global pandemic: implications for people with schizophrenia and related disorders. Schizophr Bull 46(4):752-757

Mendelson M, Nel J, Blumberg L, Madhi SA, Dryden M, Stevens W, Venter FWD (2020) Long-COVID: an evolving problem with an extensive impact. S Afr Med J 111(1):10-12 
Nemani K, Li C, Olfson M et al (2021) Association of psychiatric disorders with mortality among patients with COVID-19. JAMA Psychiat. https://doi.org/10.1001/jamapsychiatry.2020.4442

Varikuti S, Jha BK, Vopedo G, Ryan NM, Halsey G, Hamza OM, McGwire BS, Satoskar AR (2018) Host-directed drug therapies for neglected tropical diseases caused by protozoan parasites. Front Microbiol 9:2655. https://doi.org/10.3389/fmicb.2018.02655

Vidal JE (2019) HIV-related cerebral toxoplasmosis revisited: current concepts and controversies of an old disease. J Int Assoc Provid AIDS Care 18:2325958219867315. https://doi.org/10.1177/2325958219867315

Wilking H, Thamm M, Stark K, Aebischer T, Seeber F (2016) Prevalence, incidence estimations, and risk factors of Toxoplasma gondii infection in Germany: a representative, cross-sectional, serological study. Sci Rep 6:22551. https://doi.org/10.1038/srep22551

Xiao J, Prandovszky E, Kannan G, Pletnikov MV, Dickerson F, Severance EG, Yolken RH (2018) Toxoplasma gondii: biological parameters of the connection to schizophrenia. Schizophr Bull 44(5):983-992
Yelin D, Margalit I, Yahav D, Runold M, Bruchfeld J (2020) Long COVID-19--it's not over until? Clin Microbiol Infect. https://doi. org/10.1016/j.cmi.2020.12.001

Zheng M, Gao Y, Wang G, Song G, Liu S, Sun D, Xu Y, Tian Z (2020a) Functional exhaustion of antiviral lymphocytes in COVID-19 patients. Cell Mol Immunol 17:533-535. https://doi.org/10.1038/ s41423-020-0402-2

Zheng HY, Zhang M, Yang CX, Zhang N, Wang XC, Yang XP, Dong XQ, Zheng YT (2020b) Elevated exhaustion levels and reduced functional diversity of $\mathrm{T}$ cells in peripheral blood may predict severe progression in COVID-19 patients. Cell Mol Immunol 17:541-543. https://doi.org/10.1038/s41423-020-0401-3

Publisher's Note Springer Nature remains neutral with regard to jurisdictional claims in published maps and institutional affiliations. 